\title{
Assessment of Quality of As-is Building Information Models Generated from Point Clouds Using Deviation Analysis
}

\author{
Engin Burak Anil ${ }^{1}$, Pingbo Tang ${ }^{2}$, Burcu Akinci ${ }^{1}$, Daniel Huber ${ }^{3}$ \\ ${ }^{1}$ Civil and Environmental Engineering Department, Carnegie Mellon University, 5000 Forbes \\ Avenue, Pittsburgh, PA, 15213 \\ ${ }^{2}$ Department of Civil and Construction Engineering 4601 Campus Drive, Western Michigan \\ University, Kalamazoo, MI 49008-5316 \\ ${ }^{3}$ Robotics Institute, Carnegie Mellon University, 5000 Forbes Avenue, Pittsburgh, PA, 15213
}

\begin{abstract}
Three dimensional (3D) imaging sensors, such as laser scanners, are being used to create building information models (BIMs) of the as-is conditions of buildings and other facilities. Quality assurance (QA) needs to be conducted to ensure that the models accurately depict the as-is conditions. We propose a new approach for QA that analyzes patterns in the raw 3D data and compares the 3D data with the as-is BIM geometry to identify potential errors in the model. This "deviation analysis" approach to QA enables users to analyze the regions with significant differences between the 3D data and the reconstructed model or between the 3D data of individual laser scans. This method can help identify the sources of errors and does not require additional physical access to the facility. To show the approach's potential effectiveness, we conducted case studies of several professionally conducted as-is BIM projects. We compared the deviation analysis method to an alternative method - the physical measurement approach - in terms of errors detected and coverage. We also conducted a survey and evaluation of commercial software with relevant capabilities and identified technology gaps that need to be addressed to fully exploit the deviation analysis approach.
\end{abstract}

Keywords: Building Information Model, As-is, As-built, Quality Assessment, Laser Scanning, Deviation Analysis Method, Physical Measurement Method

\section{INTRODUCTION}

Government agencies and private industry engage service providers to create building information models (BIMs) of the as-built or as-is conditions of buildings and other types of facilities using laser scanners ${ }^{1}$. The process of creating an as-is BIM involves several steps, and at each step, inadvertent errors can be introduced. These errors can reduce the accuracy of the final model. Quality assurance (QA) is employed to ensure that an as-is BIM meets the project's accuracy requirements.

One method for QA of as-is BIMs is to physically measure key dimensions in the facility and compare these measurements to analogous virtual measurements in the as-is BIM [1,2]. Since it is not feasible to physically measure at every possible location, only a sampling of the measurements is obtained in practice. These measurements can be obtained using random sampling from the pool of potential measurements. Based on statistical analysis of the differences between the physical and virtual measurements, it is possible to make confidence statements about whether the model meets a given accuracy requirement.

This physical measurement approach has a number of disadvantages. In practice, measurements are conducted at a limited number of discrete locations. Therefore, critical errors can be missed because they may never be measured. Even if a component with a problem is actually measured, that measurement may be at a location that fails to identify the problem. The method also does not provide any intuition into the source of detected errors. For example, it is not possible to distinguish between a modeling error and a registration error. Finally, additional physical access to the facility may be required - in some cases, more than once, which makes the process even more time consuming.

\footnotetext{
${ }^{1}$ For brevity, we use the term "as-is" to refer to "as-built" or "as-is" hereafter.
} 


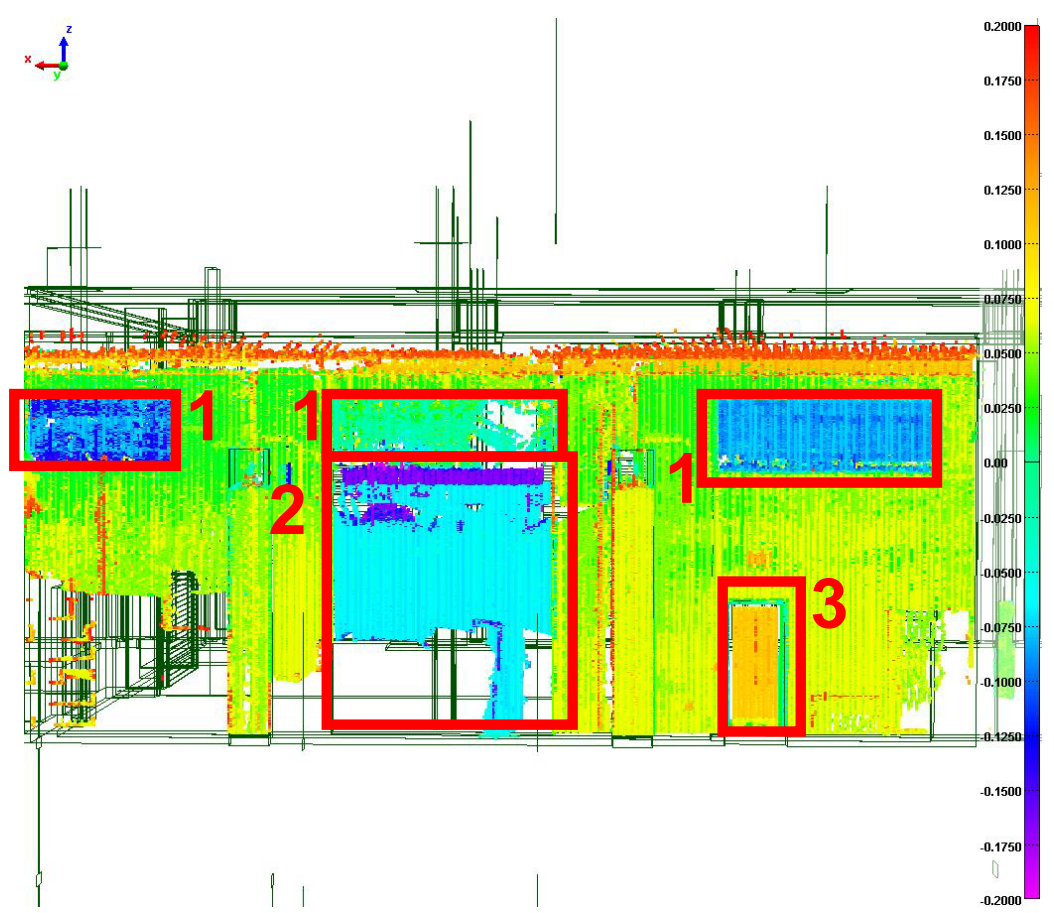

Figure 1. Deviation analysis compares points to the BIM or points to points. The figure illustrates a deviation analysis performed by comparing the points to a BIM. Problem regions found in the deviation map are highlighted by red rectangles. Regions 1 are unmodeled windows, region 2 is an indentation that was not modeled at the correct setback, and region 3 is a door that was modeled at the wrong location.

We propose an alternative method for QA of as-is BIMs created from laser scanner data that addresses the limitations of the physical measurement method. We call this approach the deviation analysis method for QA (abbreviated as "deviation analysis method" hereafter). The central idea of the deviation analysis method is that errors in the BIM can be detected by analyzing patterns in the differences between the laser scan 3D point data sets (known as point clouds) and the modeled BIM or differences between individual point clouds. In general, the as-is BIM should be in agreement with the point cloud data, and the differences (or deviations) between the two types of data should not be too large. Regions with large deviations are potential problems. Figure 1 presents the comparison of a point cloud to an as-is BIM for a wall. The legend on the right indicates the coloring scheme for the deviations based on the minimum distance of points from the wall. Regions with significant deviations that are highlighted in red rectangles are potential errors in the model. A close investigation of the deviations reveals that regions labeled as 1 are windows that were not modeled, region 2 is a setback on the wall that was not correctly modeled, and region 3 is a door with an incorrect location.

The deviation analysis method has a number of desirable properties:

- Full coverage - The method can be applied to any and every surface in a facility, including walls, ceilings, and floors.

- Ability to pinpoint error source - Different types of analysis can detect errors in different phases of the BIM creation process.

- Potential for automation - Although our current implementation is largely a manual process, there is a clear pathway for automation of the approach.

- No need for physical access or additional data - The method does not require revisiting the site to collect measurements. It is entirely self-contained within the existing data that is collected or generated in the course of the current BIM creation workflow.

- Intermediate results - It is possible to analyze the data immediately to check for potential registration or data calibration problems. Ultimately, this analysis could be conducted in the field, allowing service providers to 
recalibrate, switch to another scanner, add more fiducial targets, or retake missing or corrupted data with minimal workflow interruption.

- Ability to access entire site - Since the method is based on the laser scan data, analysis can be conducted on any part of the site that is visible by the scanner. Therefore, the method inherits the advantages provided by the ability of laser scanner to sense at long distances. These advantages include safer working conditions, shorter measurement times, and ability to measure physically inaccessible locations (such as the upper façade of a highrise building).

In order to explore the method's potential effectiveness, we conducted case studies of several professionally conducted as-is BIM projects. We compared the deviation analysis method to physical measurement approach in terms of coverage of the environment and errors detected. We also conducted a survey and evaluation of commercial software with relevant capabilities and identified technology gaps that need to be addressed to fully exploit the deviation analysis approach. This paper reports on the findings of these studies and finally discusses the future research directions required to exploit the full potential of the deviation analysis method for QA of as-is BIMs.

\section{THE DEVIATION ANALYSIS METHOD}

The deviation analysis method aims to find sources, types, and magnitude of errors in different phases of the BIM creation workflow by comparing the laser scan points to the BIM or scans to scans, and analyzing the resulting deviation maps for characteristic patterns. This idea relies on the basic assumption that the individual scans and the BIM should agree.

Deviations can be computed in a number of different ways. The simplest approach is to use the minimum Euclidian distance from a point to a surface or between two points from different scans. It is possible to take into account surface normals to obtain the sign of the deviations. Closest point correspondences can be limited based on angle tolerances with respect to the surface normals. It is also possible to compute deviations only along a given direction, such as the scanner viewing direction. In this work, we use the minimum Euclidian distance as well as the surface normals for computing signed deviations.

The method used to visualize the deviations plays an important role in revealing different patterns of deviations and, hence, different types of errors. By inspecting deviations with different visualization styles, we can expect to detect errors in different stages of the modeling process. While, a certain visualization pattern is useful for inspecting one kind of deviation, it can be less useful for another. Figure $2 \mathrm{~b}$ illustrates deviation map of a wall visualized as the signed distance of the deviations. It can be seen that the points are rotated slightly about the wall diagonal resulting in a pattern of increasing deviations in the negative direction from top left (light green region) to the bottom right (purple region). The absolute distance visualization (c) is not as useful in this case, because it hides the fact that the point cloud and the BIM are likely to be slightly misaligned. Several visualization methods can be used to inspect the deviation maps, such as binary or gradient color maps, signed or unsigned coloring schemes, or statistical analysis of the deviations. The choice of the appropriate visualization method depends on the type of the error for which the model is being analyzed. 


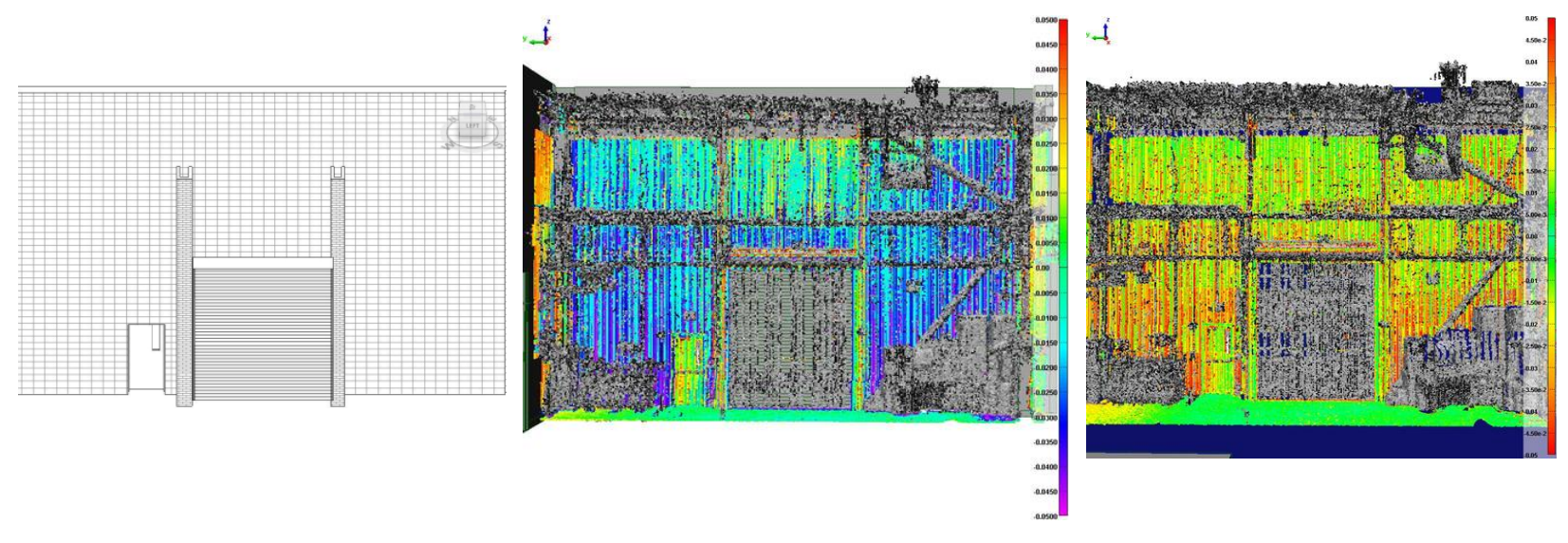

(a)

(b)

(c)

Figure 2. A deviation analysis performed by comparing the points to a BIM. (a) BIM of the wall (b) Deviation map visualized as the signed distance from the BIM. (c) Deviation analysis result visualized as the absolute distance from the BIM. Gray regions in (b) and (b) are the points that are beyond the cut-off distance.

\subsection{Types of errors that can be identified with deviation analysis method}

Generation of a BIM from point clouds involves three main stages: data collection, registration, and modeling [9]. Different kinds of errors can occur at each of these stages. The shape and magnitude of a deviation pattern can convey information indicating at which stage an error was introduced. In this paper, we primarily focus on the deviation patterns in the modeling phase because existing software already supports these capabilities and because modeling errors are more prevalent in the data that we analyzed. For completeness, we briefly described the types of patterns that can occur in the other two stages.

In the data collection phase, major sources of errors include incorrect calibration of the scanner, mixed pixels due to spatial discontinuity edges, and range errors due to specular reflections. The pattern for a calibration error would typically be similar for all scans and would appear as deviations across large portions of the data. Such patterns would not be explainable by registration errors. Figure 3 a shows a potential calibration error. The circular and wavy deviation pattern is covering the entire flat roof surface. In this case, we couldn't confirm whether this is a calibration error or an imperfection in the shape of the surface, but the fact that the circles are centered around the scanner locations indicate the higher probability of the former case.

In the data registration stage, individual scans can be misaligned. Registration errors can occur for many reasons, including incorrect localization of targets, incorrect target labeling, dynamic environments, mixed-pixels, or algorithm convergence problems. The pattern for registration error would typically appear in overlapping regions of individual scans as large deviations or as smooth variation in deviation magnitude. These patterns cannot be explained by modeling errors because they can span across different types of components. Figure $3 \mathrm{~b}$ presents a potential registration error.

In the modeling phase, registered points are converted into parametric BIM objects, such as walls, windows, doors, etc. Modeling errors can occur because modeling is currently a manual process and sometimes people make mistakes or simplifying assumptions about the geometry of the facility. In some cases, modelers may be limited by the capabilities of the modeling software. The patterns for modeling errors are typically isolated to individual components in the BIM. Modeling errors can be divided into four types, each of which has a distinctive deviation pattern:

- Failing to model a physical component: If a component is entirely missing in the model, the deviation map should show significant deviations at where the component should be. The deviation is likely to be large and may be uniform.

- Modeling a component using incorrect geometry: When modeling the facility, the modeler makes assumptions about the geometry of the building. Sometimes, these assumptions are also driven by the accuracy requirements of the modeling job. One of the most obvious modeling assumptions is that wall surfaces are 


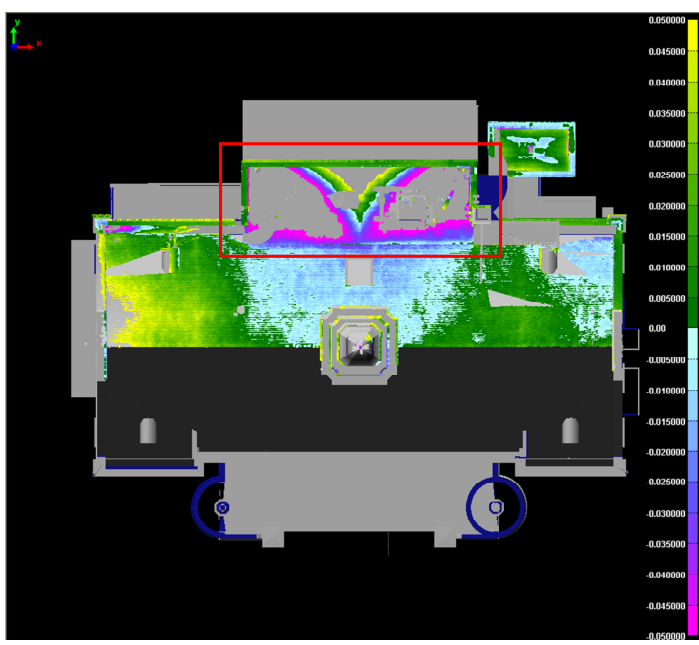

(a)

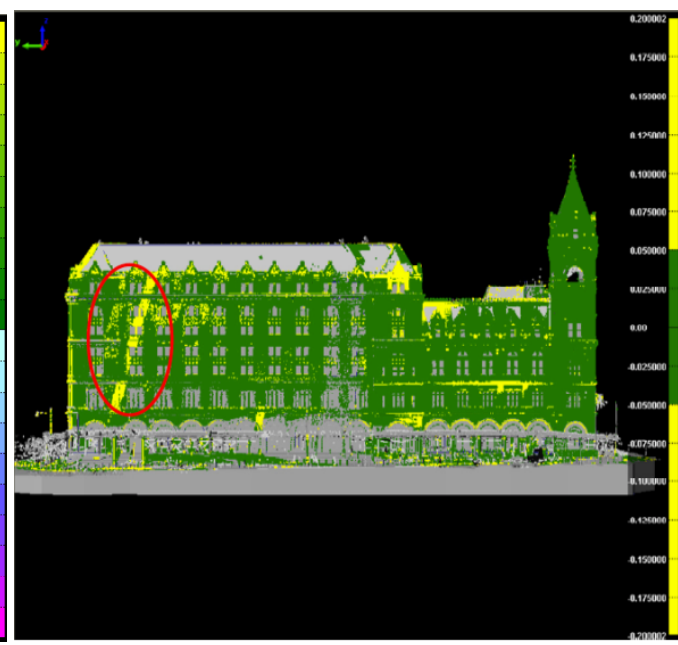

(b)

Figure 3. (a) Circular patterns indicate a potential mis-calibration. Deviations are visualized using a signed continuous color map. (b) A potential registration error causing significant deviation on the overlapping regions of the scans (The yellow stripe highlighted in red). In this binary deviation map points with deviations above $5 \mathrm{~cm}$ are yellow, points below the threshold are green, and points with no correspondence are grey.

perfectly flat and vertical, which is generally not true. Deviations from perfectly flat and vertical appear in the deviation map as gradients along the analyzed surface.

- Modeling a component using incorrect positioning: If the location of a component is incorrect in the model, it is usually possible to identify the error in the location by inspecting the deviation maps. The deviation map should show a deviation in the order of magnitude of the position error.

- Using the wrong component type to model a component: Assuming a model shape for the data is very common in modeling practice. For example, a common mistake is to assume the same window model for all similar looking windows. Differences between the actual window geometry and the assumed window geometry reveal the error. The deviation map should show the error as the difference between the correct component type and the used component type.

\subsection{Methods of deviation map visualization}

Deviations maps can be visualized in a variety of ways. The choice of visualization method depends on the goals and requirements of the QA process and also the type of error to be detected. Deviation map visualization methods can be categorized along 4 dimensions:

- Continuous vs. binary color maps: Continuous color mapping assigns a particular color value from the color spectrum to every point based on the magnitude of the deviation. In this case, deviation gradients caused by incorrect geometry assumptions and local imperfections of the model can be captured easily (Figure 2b and c). Binary color mapping sets a threshold value and colors the points differently depending on whether they are within the threshold or beyond it. This method, for example, can be used to find all deviations that are beyond 5 cm (Figure 3b).

- Signed vs. unsigned coloring: Signed deviation visualization colors the points according to their signed deviations so that the direction of the deviation with respect to the surface normal can be observed. Unsigned deviation maps color the points according to the absolute value of the deviations. This type of visualization can be combined with other types of visualizations, so that signed continuous maps, or unsigned binary maps can be produced. In our experience, signed deviation maps are more useful that unsigned maps, because they contain more information about the deviations without being a distraction. As an example, a signed deviation map can tell whether a non-flat surface is bowed inward or outward. 


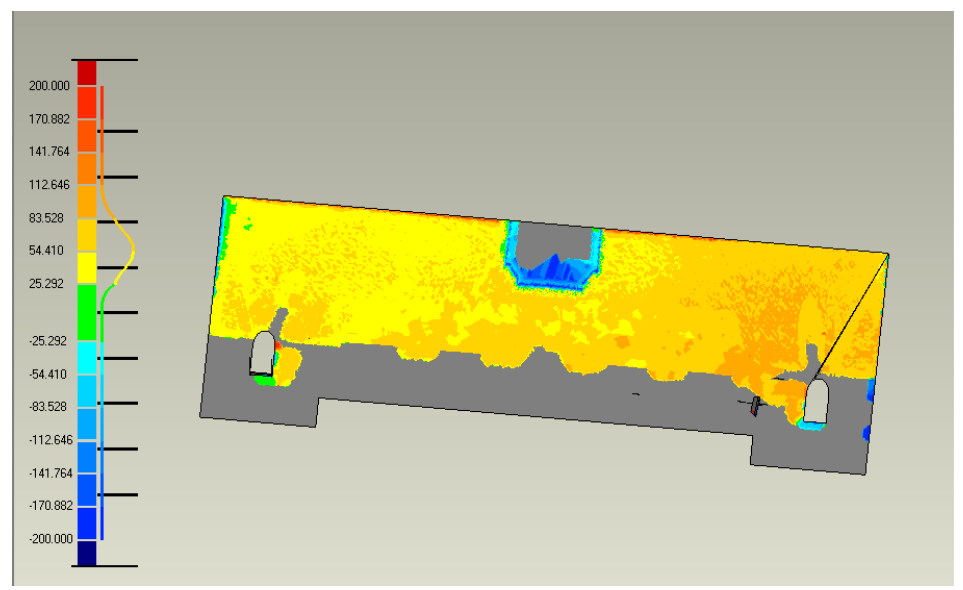

Figure 4. Histograms can give a quick sense of the deviation trend by showing the distribution of deviations. Here, the deviation on the roof of can be seen to be about $0.054 \mathrm{~m}$ in magnitude.

- Coloring points vs. coloring surfaces: The deviations can be visualized by either coloring the points or the surfaces in the model. Coloring points can detect situations where an object was not modeled at all. Coloring surfaces can detect regions where there are no points to support the model for that surface.

- Statistical analysis of the deviations: Statistical analysis can help a better understanding of the deviations. For example, in cases where multiple patterns are observed, a histogram of deviations can provide a better means of finding the magnitude of the error. In Figure 4, local deviations with orange and blue colors, as well as a dominant displacement in yellow can be observed. It is difficult to determine the magnitude of the displacement directly from the deviation map, whereas the histogram makes it easy. The histogram also indicates the spread of the deviations.

\subsection{Deviation analysis process}

The deviation analysis process for detecting modeling errors involves several steps. We assume that the as-is BIM data and the point data are already aligned. This is a reasonable assumption, since the BIM is derived from the point cloud data and should, therefore, be in the same coordinate system by default. Ideally, the entire BIM could be compared with the point data in a single operation, but Section 3 existing software is not capable of handling the large data sets that would be involved and also cannot easily visualize deviations in building interiors. To address these limitations, we segment a facility into smaller surfaces, such walls, floors, and ceilings of individual rooms, and then conduct deviation analysis separately on each surface. The data for each surface is first segmented from the as-is BIM and the point cloud data. Then, deviations are computed between the segmented BIM data and the point cloud data. Next, the deviations are visualized in the form of a deviation map. The deviation maps are then analyzed to determine the cause of each significant deviation. Finally, the results are summarized and combined with the analyses of other surfaces.

\subsection{Relation to previous work}

The idea of using 3D deviations for inspection has been used before in various contexts. We previously used a variant of deviation analysis for detecting construction defects by comparing as-built laser scanning data to the as-designed BIM [8]. The main difference of the proposed approach from our previous work and the applications in other fields is that our approach focuses on identifying the sources of errors by inspecting the patterns of deviations and on utilizing different visualization methods to capture those patterns.

The manufacturing industry uses a form of deviation analysis to inspect parts, and the ability to compute and visualize deviations has been incorporated into reverse engineering software this purpose [3]. The QA of as-is BIMs is, in many ways, a more difficult problem. First, QA for as-is BIMs is often conducted in the interiors as well as the exteriors of a facility. Existing manufacturing inspection software does not work well for visualizing and inspecting interior regions. Second, BIMs are segmented into components, and it is therefore necessary to reason about the deviations at the component level, which is not needed in manufacturing inspection. Finally, surfaces of interest in the laser data are often 
occluded by equipment or furniture, which makes the analysis more challenging. In manufacturing, occlusion occurs less frequently because it is a controlled environment.

The deviation analysis method to QA is related to the process of clash detection. Clash detection is used in CAD-based projects to determine whether a modification to a facility will interfere with the existing structures in the facility. Clash detection can also be used with laser scan data. In such a case, a CAD model of the new components is checked against the point cloud to determine if any points in the point cloud fall inside or too close to the surface of the CAD model. If such an event occurs, it is declared a "clash," and the clashing part of the model is highlighted, usually in red.

Clash detection can potentially be used for performing QA by comparing the point cloud data to the as-is BIM. However, clash detection is an imperfect tool for deviation analysis for several reasons. First, the tools were not designed for such a purpose, so it can be cumbersome to interpret the results. For example, since the clash detection tool reports only clashes, it is difficult to identify small parts of the model that do not clash. Yet these small parts are exactly the components of interest for QA, since those are the potential modeling errors. Clash detection is also normally implemented as a binary clash/non-clash decision. Therefore, it is more difficult to analyze the degree of deviation. The deviation analysis method can be seen as the generalization of the clash detection method, where the deviations of individual points are reported and colored deviation patterns are inspected to identify the source and type of the errors.

\section{APPLICABILITY OF DEVIATION ANALYSIS WITH CURRENT SOFTWARE TOOLS}

We conducted a survey and evaluation of the capabilities of existing software in order to explore the applicability of deviation analysis method with the existing tools and potential bottlenecks. In addition, this survey helped us to understand the commonalities of the software and features of existing software in applying the deviation analysis procedure that needs to be improved. We identified an initial list of 29 software packages and narrowed down the list to 5 packages that can perform deviation analysis: Polyworks (Innovmetric) [3], Rapidform (Inus Technology) [5], Geomagic (Geomagic) [4], and Cyclone (Leica) [6]. Additionally, we evaluated Navisworks (Autodesk) [7], which is able to perform clash detection between CAD models and laser scan data, in order to better understand the capabilities and limits of the clash detection approach. We then performed an in depth evaluation of the software using criteria that we derived from deviation analysis requirements. We organized these criteria into three categories:

- Deviation measurement and visualization: The software should be able to perform deviation analysis by computing distances of points-to-surfaces or points-to-points. In addition, the software should be able to visualize the deviations in a variety of ways in order to inspect the deviation patterns and identify errors.

- Interoperability: Deviation analysis involves two types of input data files - point cloud data and as-is BIM data files. There is no established standard for point cloud, though the ASTM E57 committee has recently approved a standard point cloud data format [10]. One widely accepted data exchange format for BIMs is the Industry Foundation Classes (IFC) format. The ability for software to correctly import data in a useful format is necessary for supporting the actual deviation analysis processing.

- Performance on large data sets: The raw, as-is data for a typical facility can contain hundreds of millions or even billions of points. As such, it is important for analysis software to be capable of handling extremely large data sets. The data sets we used for evaluation were smaller, with up to seven scans and up to twenty million points. Even with this smaller size, we still observed some software problems in handling the data.

We evaluated each package using a subset of the data from an entire project. In our case study, the as-is BIM data was provided in Autodesk Revit format, and the point cloud data was provided in a Leica Cyclone database. We followed the process described in Section 2.3 to perform deviation analysis on the sample. In our evaluation process for each package, we began by testing the import formats. We tried exporting from the native format of the point clouds and the BIMs to the formats accepted by the software package and then importing them. None of the software packages were able to import our data files directly. In all of the cases, we had to find appropriate file formats for data exchange, both for the point clouds and the BIMs. Next, we evaluated the capabilities of the software to compute and visualize deviation maps with the various methods described above. For all of these steps, we tracked the time required to perform each operation and noted any problems that occurred. These observations were used to evaluate the performance capabilities on large data sets. 


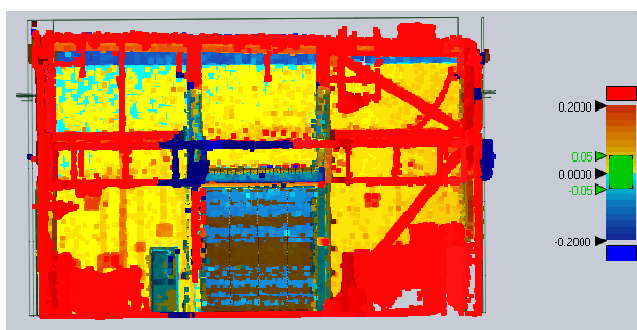

(a)

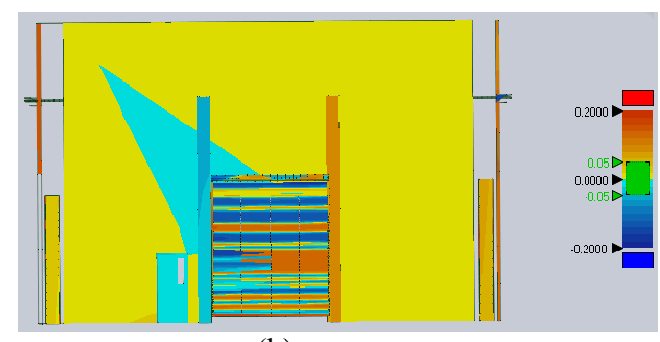

(b)

Figure 5. Coloring points and coloring surfaces gives different results. With the point cloud colorization in (a), a deviation in the wall surface can be seen in the upper left corner (light blue region). The red regions are due to clutter. With the CAD model colorization in (b), the deviation in the upper left corner is lost, and artifacts, such as the large blue triangle, are introduced. The effect is caused by the deviation computation algorithm used by the software, which appears to average the deviation over entire polygons in the underlying mesh.

The software evaluation and comparison study provided valuable insight into the capabilities and limitations of currently available software in performing the deviation analysis method in the sense that we are proposing in this paper. The following paragraphs discuss the findings of the evaluation.

\subsection{Deviation measurement and visualization}

In the previous section, we discussed that there are a number of ways for computing and visualizing deviations. We discovered limitations of the software that constrain the usage of these software packages for the deviation analysis method. Particularly, coloring of surfaces instead of points and working in the interiors of the models had been observed to need attention.

Some of the software packages support coloring surfaces in the CAD model (in addition to coloring individual points). In those implementations, the coloring of the surfaces is achieved by averaging the comparison results over entire polygons in the underlying polygonal mesh surfaces. This approach can lead to misleading conclusions because the method can distribute the deviations over an area different in size than the actual deviation, as illustrated in Figure 5. The point-based colorization in Figure 5a shows a deviation pattern with negative values around the top left of the wall (blue regions) and increasing pattern towards the bottom right (yellow and orange). This pattern may indicate a registration error. The red colored points are beyond the cut-off distance of $20 \mathrm{~cm}$. Figure $5 \mathrm{~b}$ shows the same deviation, this time with the surfaces colored. In this case, the deviations are not visible. Artifacts, such as the large blue triangle, are introduced into regions where there should be no significant deviations. It is likely that these software packages assume that the surfaces will be made up of very small polygons, in which case this problem would be less apparent.

Another drawback we observed was related to visualization of interiors of the models. The software packages we tested were not specifically designed for inspecting buildings or models that have complex interior surfaces. In order to perform deviation analysis, however, working in the interiors is a necessity. The result is that navigating inside the buildings was cumbersome and sometimes impossible. We found a workaround by clipping of the sections of the buildings and isolating the regions to analyze, but this procedure is more time consuming.

These limitations can mostly be attributed to the implementation of the algorithms and can be addressed by implementing methods that take the discussions in this section into account. For instance, a better way to implement surface-based deviation coloring would be to use a texture map to enable high-resolution deviation coloring within each polygon. 


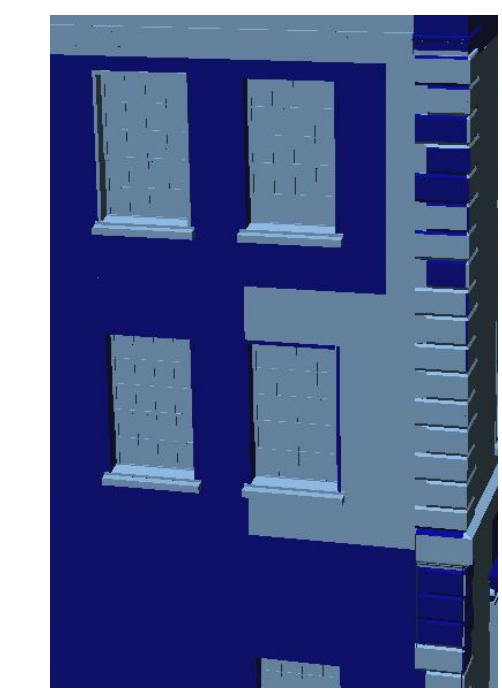

(a) Orientation of the surfaces

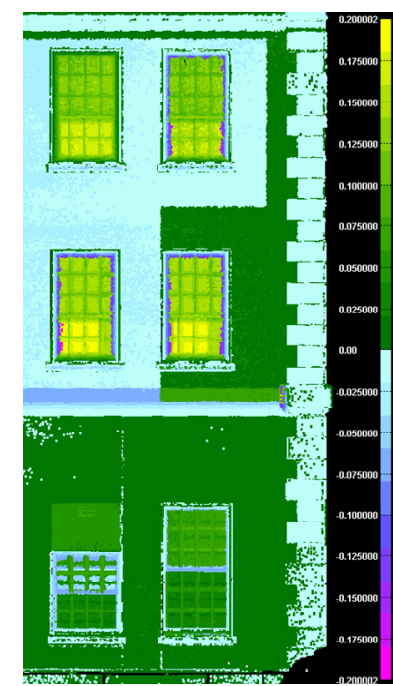

(b) Deviation analysis visualization

Figure 6. Data import causes some surfaces in the model to flip, as shown in (a), where positive faces are colored grey and negative faces are colored blue. Ideally, all of the surfaces should be either blue or grey. The result is inconsistent visualization patterns in the deviation analysis, as shown in (b). Note the sharp boundary near the center window on the right.

\subsection{Interoperability}

None of the tested software supports industry standard BIM formats, such as IFC, or commercial BIM formats, such as Autodesk Revit files. It was necessary to use various intermediate formats to downgrade the BIM data to a CAD format that is readable by the point cloud processing software. This limitation restricted us to a very small number of formats or required complex sequences of data transformations.

Some issues arose even when the data import was successful. One significant issue revolves around how surface normals in the as-is BIM are maintained. In the deviation analysis, the color of the deviation in visualization can depend on the sign of the deviation with respect to the surface normal. Therefore, it is important to maintain the correct surface normals for the model surfaces. We observed that during the conversion process, sometimes the surface normals were reversed (Figure 6).

Another observation was that one of the tested software packages organized the points into a grid during import, which ultimately altered the 3D coordinates of the points. We found that by using a different file format, the conversion can be avoided. For deviation analysis, the points that were used to model the facility and the points that are used as the ground truth for the deviation analysis should be the same. Therefore, import processes that result in such conversions should be avoided if possible.

\subsection{Large data sets}

Due to software limitations, working with large data sets is not very easy and often requires the data to be divided into pieces for analysis. With our data sets, which contained up to seven scans and up to 20 million points, we experienced software crashes during import, registration, and deviation computation. We tried to identify practical limits for the data size to avoid crashes. This trial and error process involved analyzing a small number of points at the beginning and increasing the number of points by small amounts until the software crashed. For some software packages, we were able to identify a consistent practical limit on the size of the data, whereas for others, a practical limit could not be achieved. Thus, for those packages, the user would need to determine the practical file size limit for every analysis by trial and error, which affects the user experience and the time required to obtain analysis results. 


\section{COMPARISON TO THE PHYSICAL MEASUREMENT METHOD}

We objectively evaluated the deviation analysis method by comparing it side by side with the physical measurement method on a case study of a professionally modeled commercial building. In separate research, the National Institute of Standards and Technology (NIST) conducted an analysis using the physical measurement method [2]. Researchers from NIST (along with a researcher from our group) collected 285 measurements throughout a facility using either a laser distance meter and in some cases, a measuring tape. Most measurements were wall-to-wall or floor-to-ceiling dimensions of the rooms. Some were object dimensions, such as window size, other measurements included distances between objects (e.g., column to wall distance) and circumferences of pipes. Multiple measurements were conducted at different locations on the object, and the results were averaged.

The NIST study revealed several challenges specific to the physical measurement approach. First of all, a number of the measurements were deemed to be incorrect, and, a second visit to the site was necessary in order to double check the manual measurements that did not agree with the corresponding BIM measurements. This observation emphasizes that physical measurement itself is not $100 \%$ reliable. Furthermore, the laser distance meter used for physical measurement could not measure some of the longer dimensions, and it was necessary to rely on a measuring tape, which is a less accurate method. The NIST researchers also observed that physical obstructions prevented some of the measurements from being obtained.

We compared the deviation analysis method to the physical measurement method in two ways. First, we tested whether the two methods agree on common measurements. Second, we evaluated the relative coverage of the two methods.

\subsection{Comparison on Common Measurements}

Among the different types of measurements, the room dimension measurements are the best suited for comparison between the two approaches. We identified all such measurements for the rooms of the facility. Since the deviation analysis method measures absolute deviations of object position, while the physical measurement method measures relative distance between two surfaces, it is not possible to compare the measurements directly. Therefore, we created virtual relative measurements in the deviation maps at the same locations that were used for the physical measurement method (Figure 7). For example, if the physical measurement method measured the distance between the east and west walls of a room at a specific location, we used the deviation maps of those same walls to determine the absolute offsets of each wall, then we subtracted the offsets on opposing walls to determine the relative distance. We then compared these derived measurements with the room dimension error obtained by the physical measurement method. We not only considered measurements that were outside of the specifications of the requirements (out-of-spec), but we also compared our method to the physical measurement method for measurements that were within the specified tolerances (in-spec). 


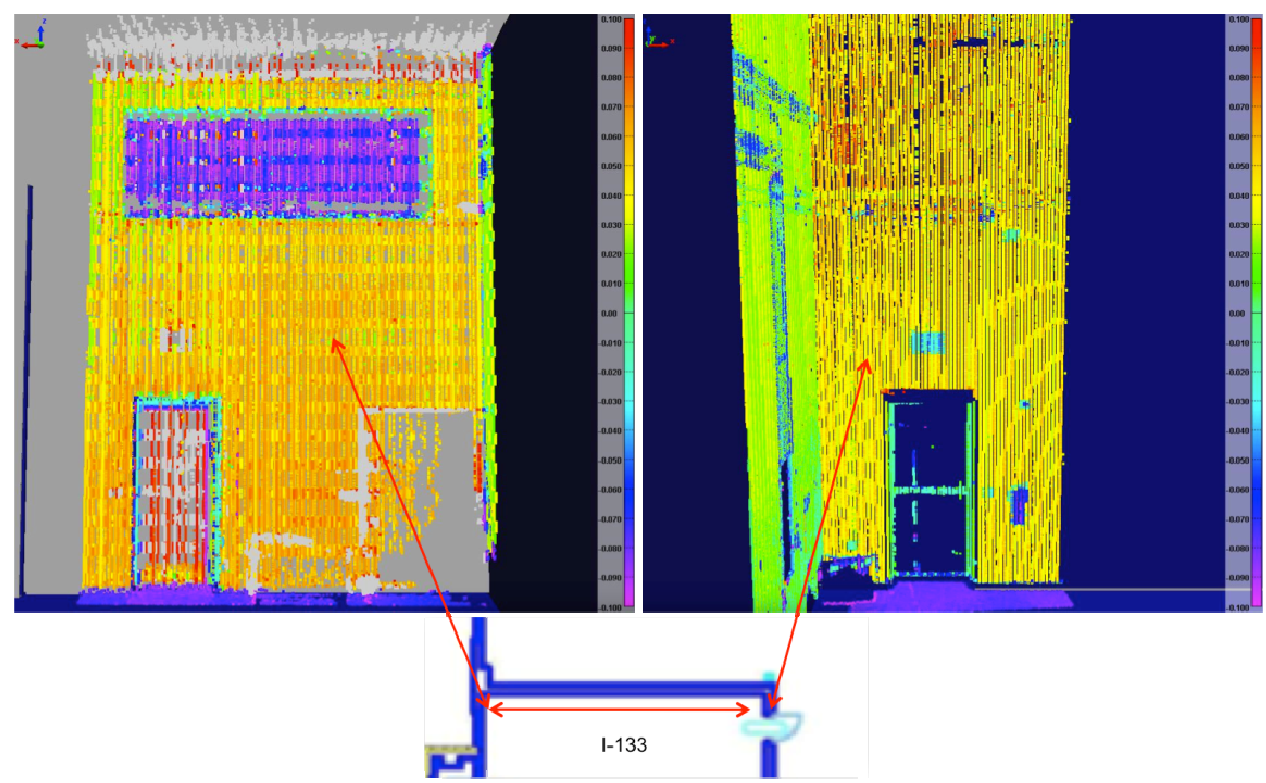

Figure 7 Equivalent relative measurements were obtained for room dimensions by subtracting the relative offsets of the deviations for the walls as shown in the plan view (bottom). First, deviation maps were obtained at locations where physical measurements were taken (top left and right). Then, by subtracting the offsets on these two walls, an equivalent measurement was obtained.

Table 1. Summary of number of errors detected by the physical measurement method and the deviation analysis method.

\begin{tabular}{|c|c|c|c|c|}
\cline { 2 - 5 } \multicolumn{1}{c|}{} & \multicolumn{2}{c|}{ Physical measurement method } & \multicolumn{2}{c|}{ Deviation analysis method } \\
\hline Room & $\begin{array}{c}\text { \# } \\
\text { measurements }\end{array}$ & $\begin{array}{c}\text { \# errors } \\
\text { identified }\end{array}$ & $\begin{array}{c}\text { \# interest } \\
\text { regions }\end{array}$ & $\begin{array}{c}\text { \# errors } \\
\text { identified }\end{array}$ \\
\hline Room 1 & 13 & 4 & 6 & 17 \\
\hline Room 2 & 11 & 5 & 3 & 6 \\
\hline TOTAL & $\mathbf{2 4}$ & $\mathbf{9}$ & $\mathbf{9}$ & $\mathbf{2 3}$ \\
\hline
\end{tabular}

We found that the two methods agreed with one another in terms of whether the measurements were in-spec or out-ofspec with respect to the accuracy threshold. Among 24 measurements we compared, nine measurements conducted by the NIST team referenced components that were not modeled in the BIM. The remaining fifteen measurements agreed within $1 \mathrm{~cm}$ except for one measurement. For the disagreeing measurement, we found a significant error in the measurement $(45 \mathrm{~mm})$ whereas the physical measurement method did not find a significant error $(5 \mathrm{~mm})$. There are a number of possible explanations for this difference. It is possible that either the virtual measurement was conducted by NIST using an updated BIM or that either the physical or BIM measurements was recorded incorrectly. It is also possible that the measurements are all correct, but that they were taken at a slightly different location.

\subsection{Coverage Comparison}

Since the two methods have different concepts of coverage, it is difficult to quantify coverage in an objective manner. The physical measurement method makes sparse measurements throughout a room. If three points along a ten meter wall are measured, what "coverage" does that equate to? Instead of computing coverage directly, we developed an approach that measures coverage indirectly through a comparison of the number of modeling errors detected by each method. Assuming that the modeling errors are distributed uniformly throughout the site, the ratio of the number of errors detected by each method should give an approximation of the relative coverage achieved by the two methods. Using this approach, we computed the number of errors detected by the two methods in a sampling of rooms on the site (Table 1). The physical measurement method identified nine significant modeling errors, whereas the deviation analysis method identified 23, which is 2.5 times more. This suggests that the physical measurement method is effectively achieving $40 \%$ coverage. 


\section{DISCUSSION AND CONCLUSIONS}

Our evaluation of the deviation analysis method for QA indicates that the method can be used to reliably identify modeling errors throughout a facility. The comparison with the physical measurement method shows that the deviation analysis approach has numerous significant advantages, the most prominent of which is complete coverage. The deviation analysis method provides complete coverage of the surfaces in a facility, whereas the physical measurement method only allows sparse measurements. The result is that the deviation analysis method can identify numerous errors that would be missed by the physical measurement method. For example, both walls in Figure 7 show slightly different values of deviation from the rest of the wall surfaces. Both the physical measurement method and the deviation analysis method found the same magnitude of deviation from the correct wall location, but only the deviation analysis method discovered the deviation of surface geometry from the actual. While the physical measurement method may be able to offer statistical guarantees of conformance to an accuracy specification, the deviation analysis method can provide deterministic guarantees that a model completely represents the underlying data with a given accuracy specification.

Another benefit of the deviation analysis method is that it computes absolute measurements rather than relative measurements. The measurements in the physical measurement method are typically relative measurements, such as the distance between two walls. While it is possible to convert two absolute measurements into a relative measurement, the reverse process - converting a relative measurement into absolute measurements - is not possible. Therefore the deviation analysis method measurements are a more general and more flexible type of measurement. This feature of the deviation analysis method is useful because, it does not depend on the existence of another component or prior knowledge about the physical environment in order to perform the measurements.

The sparse nature of the physical measurement method prevents certain types of modeling errors from being detected even if physical measurements were obtained for the surface. For example, if a wall is slightly rotated from its true orientation, the physical measurement method may find that the average wall position (at least relative to another wall) is correct. If a different measurement location were chosen, the error may have been detected. Even if the entire surface is within the accuracy specifications, it is potentially useful to know that the alignment is slightly incorrect.

\section{FUTURE WORK}

Based on our software comparison study, existing software is not very well suited to the task of deviation analysis. The existing software was designed for other purposes, and using it for this type of analysis does not necessarily lead to the best results. However, we are not aware of any other options at this time that are better than the software we tested. There are a number of software technology gaps, including interoperability issues, limits on data size, and constraints on the visualization. These gaps suggest one direction for future research. It is possible to clearly document the software technology gaps and then develop new software or work with software vendors to improve their software capabilities in order to enable the industry to benefit from the method.

Other potential directions for future research are related to improving the process by developing computer vision algorithms to automate the procedure, investigating the deviation patterns of other types errors to expand the applicability of the method, and investigating other potential uses of the deviation analysis method. First, we can develop methods to automate or semi-automate the inspection process. Even the underlying process of identifying and extracting the significant regions of interest could be easily automated. All of these advances would ultimately lead to faster, more reliable, and more comprehensive generation of deviation analysis reports.

Another area that needs further development is the analysis of registration and calibration errors using the deviation analysis method. We have not focused as much on this aspect of the method because the software tools for this process are not as well developed. However, the idea has much promise, and it can potentially identify errors early in the process, leading to significant cost savings in the overall BIM creation process.

A third path for future research is to extend the types of deviations that can be detected. The current deviation maps are best suited for detecting variations perpendicular to a surface. Deviations within the plane of a wall surface are more difficult to identify. For example, capturing errors in the width doors requires a close inspection around the door frame. Therefore, special attention needs to be paid to measuring deviations in the widths of objects in the region of interest. 


\section{ACKNOWLEDGEMENTS}

This material is based upon work supported by the U.S. General Services Administration under Grant No. GS00P09CYP0321. We thank Geraldine Cheok and NIST for making their research data available to us.

\section{REFERENCES}

[1] Cheok, G.S., Filliben, J.J., Lytle, A.M., Guidelines for accepting 2D building plans, NIST Interagency/Internal report (NISTIR)- 7638 (2009)

[2] Cheok, G.S., Franazsek, M., Phase III: Evaluation of an Acceptance Sampling Method for 2D/3D Building Plans, NISTIR 7659, (2009)

[3] Innovmetric, Inc., Polyworks v9.0, www.innovmetric.com, Last accessed October 2010

[4] Geomagic, Inc., Geomagic, www.geomagic.com, Last accessed October 2010

[5] INUS Technology, Inc., Rapidform, www.rapidform.com, Last accessed October 2010

[6] Leica, Inc., Cyclone, http://hds.leica-geosystems.com/en/Leica-Cyclone 6515.htm, Last accessed October 2010

[7] Autodesk, Inc., Navisworks, http://usa.autodesk.com/adsk/servlet/pc/index?siteID=123112\&id=10571060, Last accessed October 2010

[8] Yue, K., Huber, D., Akinci, B., Krishnamurti, R., The ASDMCon Project: The Challenge of Detecting Defects on Construction Sites, 3D Data Processing Visualization and Transmission, International Symposium on, Third International Symposium on 3D Data Processing, Visualization, and Transmission (3DPVT'06), 1048-1055 (2006)

[9] Tang, P., Huber, D., Akinci, B., Lipman, R., Lytle, A., Automatic reconstruction of as-built building information models from laser-scanned point clouds: A review of related techniques, Automation in Construction, 19(7), 829$843,(2010)$

[10] Huber, D., "The ASTM E57 File Format for 3D Imaging Data Exchange," in Proceedings of the SPIE Vol. 7864A, Electronics Imaging Science and Technology Conference (IS\&T), 3D Imaging Metrology, San Francisco, CA 2011 (in publication) 